\title{
Test and Improvement of Flow Distribution in a Sudden Changed Structure
}

\author{
Xiaozhang Zhang ${ }^{1, *}$ and Bin Chen ${ }^{2}$ \\ ${ }^{1}$ Department of Engineering Physics, Tsinghua University, Beijing, P R China \\ ${ }^{2}$ Shanghai Starse Instrument, Shanghai, P R China \\ *Corresponding author
}

\begin{abstract}
In this paper, we describe the work on measuring flow distribution down-stream of a $90^{\circ}$ elbow, and on improving the flow to satisfy installation of a flow meter. In the sudden changed flow channel, flow velocity distribution is very irregular. Both asymmetry and swirl exist, which cause severe error in flow rate measurement. To improve the flow, a honeycomb with distributed blocks was used. The result showed that this design is able to largely improve the flow distribution.
\end{abstract}

Keywords-internal flow; velocity measurement; straightener design

\section{INTRODUCTION}

Flow meters are widely used in many fields such as industry, civil supply, commercial trade and environment monitor etc. When a flow meter is installed onto a pipe line, some conditions have to be fulfilled. For example, certain length of straight upstream pipe is needed to ensure the flow into the flow meter being fully-developed This is important, because some flow meters are very sensitive to flow velocity distribution. For instance, a pure swirl in pipe does not contribute to flow rate but certainly drives a turbine turning faster, which make reading error in a turbine flow meter. Engineers working in the field of flow measurement always seriously maintain reasonable conditions when install flow meters in pipe line. There are technical standards to follow [1].

However, in many cases in application, certain place has to have a flow meter but the installing standard conditions can never be obtained. In this case technology of flow modification is used.

In this paper, we report our work on improving flow distribution immediate after a $90^{\circ}$ elbow. We made a specially designed structure to improve the flow channel. Flow distributions before and after the improvement were measured. It was shown that by introducing a specially designed honeycomb straightener, flow right after the $90^{\circ}$ elbow could be modified to reasonable satisfied rectlinear and flat flow. This improvement made it possible for a flow meter to be installed right after the elbow.

\section{EXPERIMENT}

Owing to some particular circumstance, the pipe turns a sharp $90^{\circ}$ without a smooth transition in between. Moreover, the pipe diameter is also changed to a sudden reduction (See
Figure 1 for the structure). Thus, up stream flow will hit at the wall facing to it and is forced to go into the smaller tube vertically up. But inertia will make the flow velocity reflect in pipe wall. Thus severe asymmetry happens in the vertical flow. Moreover, strong swirl also exits. All these make the vertical flow in the smaller tube being very complicated, where the flow rate is supposed to be measured. This is certainly not suitable for a flow meter.

Our task is to make the flow in the vertical tube to be rectlinear and uniform, so that flow measurement inside the tube will have a good accuracy.

The experiment was completed in an air flow calibration installation (See Figure 2 for the experiment section). Optical method met difficulty because outside the vertical tube there was flow as well. We open the up part of the vertical tube letting the flow just go into the air, so that Pitot tube can insert into the tube

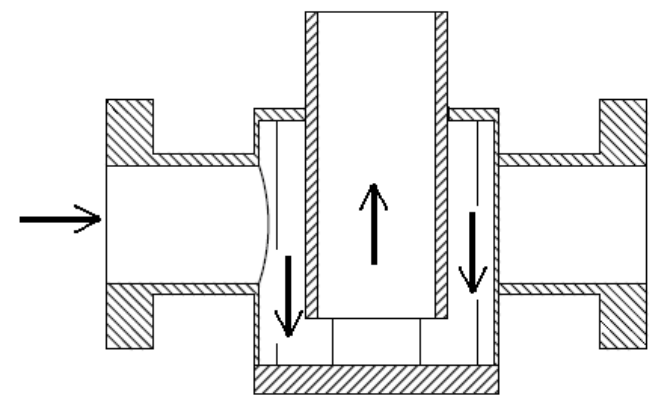

FIGURE I. STRUCTURE OF FLOW CHANNEL

The Pitot tube is $2.5 \mathrm{~mm}$ in diameter, while the measured tube ( the vertical one in Figure 1) has a diameter of $50 \mathrm{~mm}$. This means that influence of the Pitot tube in most positions can be ignored[2]. Also, calibration is done of the Pitot readings in the same flow rig by standard hot-film velometer.

The cross section of the measured tube were divided by $20 \times 20$ net grid (coordinates of $x$ and $y$ ). Three positions along the tube axis were taken (coordinate of $\mathrm{z}$ ). These are $0 \mathrm{~mm}$, $30 \mathrm{~mm}$ and $100 \mathrm{~mm}$ from the up exit.

The coordinates of the measurement points were assured by a precise $3-\mathrm{D}$ coordinator. ( See the right in Figure 2). 


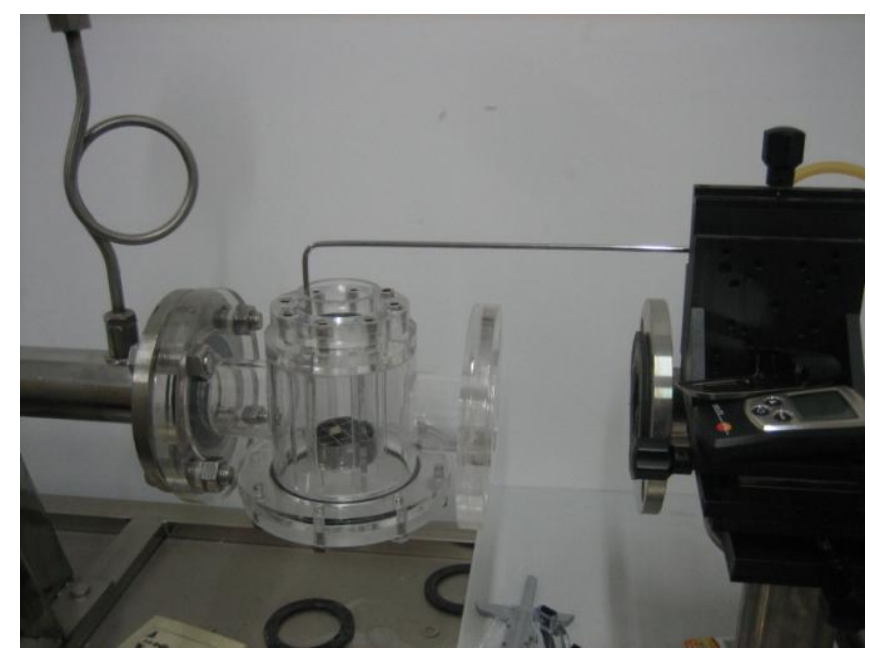

FIGURE II. PHOTO OF EXPERIMENT SYSTEM

\section{RESULTS AND CONCLUSIONS}

The first set of the flow field data without any improvement was very irregular. See Figure 3 for example. Then a honeycomb flow straightener was designed to install at the inlet of the vertical tube. We found that it largely made the flow straight. But non-uniform distribution was still considerable (See Figure 4). After careful observation of the flow distributions, we then block some selected holes of the honeycomb straightener. Basic concept for us was to block the positions where flow velocity was large then elsewhere. But the job needed to try and measure for several times before satisfied result was obtained. We finally reached to reasonable good flow distribution. Figures 5 shows the honeycomb pattern where grey color means blocked holes.

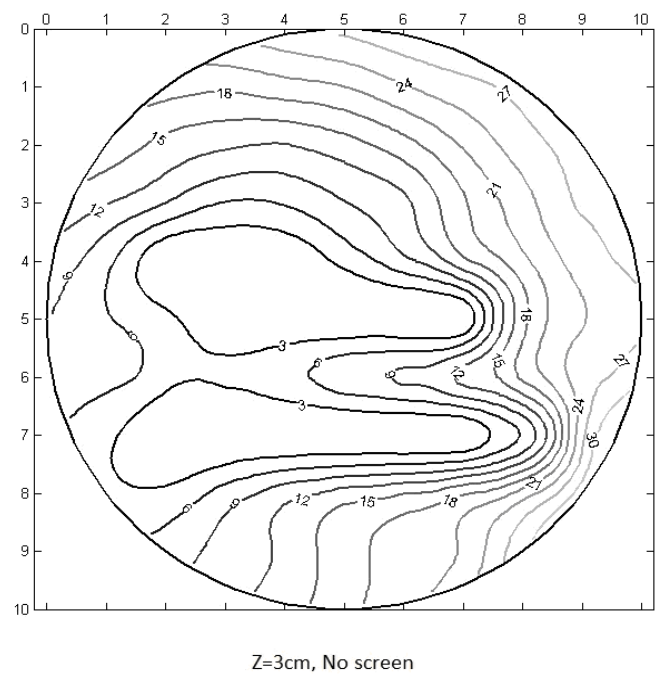

FIGURE III. ORIGINAL FLOW

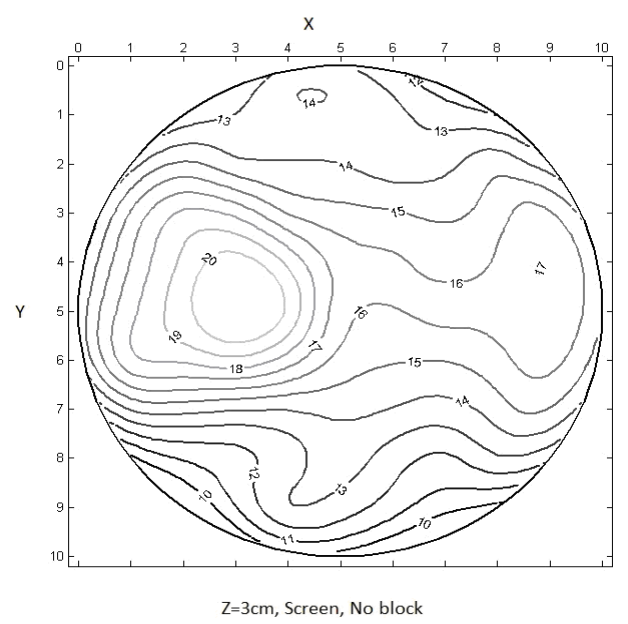

FIGURE IV. FLOW WITH HONEYCOMB

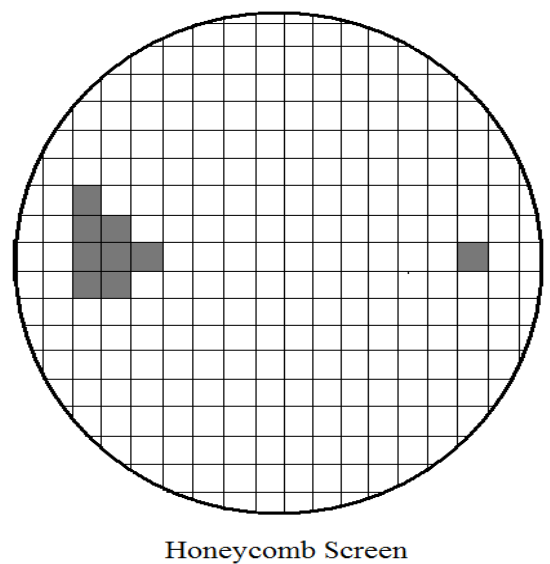

FIGURE V. HONEYCOMB WITH DISTRIBUTED BLOCKS

Figure 6 shows one of the flow distributions in the axis position of $30 \mathrm{~mm}$ up stream of the measured tube exit.

We also tried to find if the degree of swirl in the exit of the tube using hot-film velometer. The measurement was very difficult. The data were not reliable. So we would not show them here. However, we think that swirl will largely die out if the axis dimension of the honeycomb is long enough.[3] of

To further evaluate the results, we appoint a velocity ratio

$$
\frac{V_{\text {Max }}}{V_{\text {Mean }}}
$$

where

$$
V_{\text {mean }}=\frac{1}{N} \sum_{i=1}^{N} V_{i}
$$

as a measurement of the flatness of flow velocity in the tube. 
The calculated ratios were shown in Table 1, where fully developed laminar flow velocity distribution can be theoretically obtained. For fully developed turbulent flow, we use Nikuradse's empirical formula. For measurement data, the mean velocity is calculated from the direct sum of measured data, divided by the number of the measured points using Equation (2). From Table 1 we can see that the original flow velocity distribution in the measured tube has a velocity ratio of 2.2:1, which means very irregular. After installing a honeycomb at the inlet of the measured tube, the ratio becomes 1.4:1. This means the flow distribution become flat. Further improvement is obtained by blocking chosen holes of the honeycomb.

It should be noted that an ideal flat velocity in the tube does not mean the best condition for a flow meter. However, since most of industrial flows are turbulent, whose distributions are flat compared to laminar flows. In our case, the typical flow velocity is $10-15 \mathrm{~m} / \mathrm{s}$, this is turbulent. Considering this, we choose flat flow velocity distribution as a good condition for a flow meter. Meanwhile, there still needs an evaluation of the flow meter's reading uncertainty because this situation is not a standard.

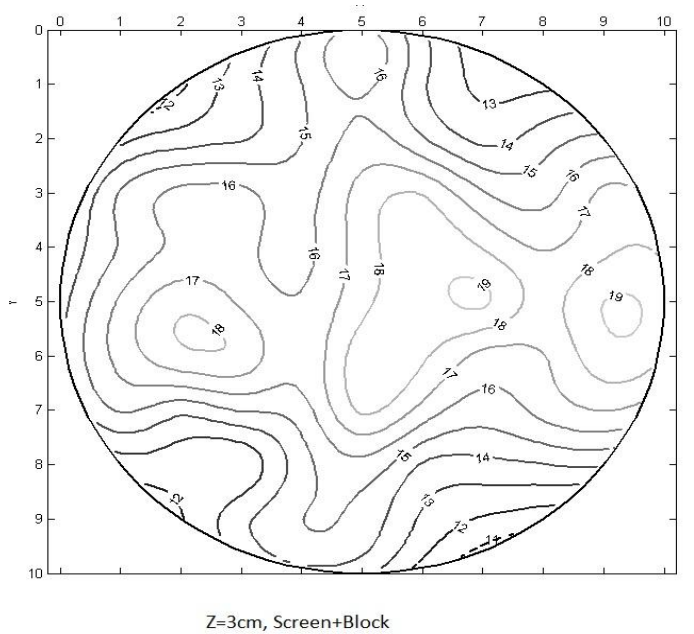

FIGURE VI. FLOW OF FINAL IMPROVEMENT

TABLE I. VELOCITY RATIOS IN VARIOUS FLOW CONDITIONS

\begin{tabular}{|c|c|}
\hline Flows in various conditions & $\mathbf{V}_{\text {Max }} / \mathbf{V}_{\text {Mean }}$ \\
\hline Fully developed laminar & $2: 1$ \\
\hline Fully developed turbulent & $\sim 1.2: 1$ \\
\hline Original & $2.2: 1$ \\
\hline With honeycomb & $1.4: 1$ \\
\hline With Honeycomb+blocks & $1.2: 1$ \\
\hline
\end{tabular}

We conclude that the designed honeycomb with specially blocked holes is a practical way to obtain uniform flow.

\section{REFERENCES}

[1] ISO 5167: Specification for square-edged orifice plates, nozzeles and venturi tubes inserted in circular crossed-section conduits running full.

[2] Baker R C: Flow Measurement Handbook, Cambridge Univ. Press, 2000

[3] Kinghorn F C et al : The use of etoile flow straignteners with orifce plate in swirling flow, J. Flow Measurement and Instrumentation, 1991, N2 pp162-8 